\title{
Selective Area Nd:YAG Laser Functionalization of Digital Photocorrosion GaAs/AlGaAs Biosensor
}

\author{
Hakim Grib $^{1,2}$, Khalid Moumanis ${ }^{1,2}$, Walid Mohamed Hassen ${ }^{1,2}$ and Jan J. Dubowski ${ }^{1,2}$ \\ ${ }^{1}$ Laboratory of Quantum Semiconductor and Photon-based BioNanotechnology, Department of \\ Electrical and Computer Engineering, Interdisciplinary Institute for Technological Innovation \\ (3IT), Université de Sherbrooke, 3000 boul. de l'Université, Sherbrooke, J1K0A5 Québec, Canada \\ ${ }^{2}$ Laboratory of Nano-technologies and Nano-characterization (LN2) - CNRS UMI-3463, Interdisci- \\ plinary Institute for Technological Innovation (3IT), Université de Sherbrooke, 3000 boul. de l'Uni- \\ versité, Sherbrooke, J1K0A5 Québec, Canada \\ E-mail: jan.j.dubowski@usherbrooke.ca
}

\begin{abstract}
Digital photocorrosion (DIP) of GaAs/AlGaAs nanoheterostructures has been found sensitive to semiconductor surface states and, thus, attractive for rapid detection of negatively charged bacteria in aqueous environment. However, calibration of DIP biosensors depends on the reproducibility of chipto-chip surface properties that are related to the chip fabrication process. To reduce the error related to this characteristics and provide a reference signal, we have examined the fabrication of biochips with a selective area removed biofunctionalization layer. The GaAs/AlGaAs biochips were coated with 1mM 16-mercaptohexadecanoic acid (MHDA) self-assembled monolayers (SAM) designed for interfacing antibodies suitable for immobilization of bacteria. Selective area thermal desorption of SAM was investigated with an Nd:YAG laser emitting at $1064 \mathrm{~nm}$ in a continuous wave mode. The exposure of a biofunctionalized and laser-processed chips to bacterial solution resulted in a selected area capture of bacteria. With a low-cost light emitting diode, this approach should allow the realization of an advanced DIP biosensor with a small surface area designed for referencing the photocorrosion process of a biosensor.
\end{abstract}

DOI: $10.2961 /$ jlmn.2020.02.2001

Keywords: digital photocorrosion biosensor, GaAs/AlGaAs nanoheterostructures, self-assembled monolayers, thermal desorption, internal referencing, Nd:YAG laser, bacteria biosensor

\section{Introduction}

Digital photocorrosion (DIP) biosensors have been investigated for rapid detection of Escherichia coli $[1,2]$ and Legionella pneumophila [3, 4] bacteria. The simplicity of this approach, the low cost of an optical reader and the potential to reuse the biochips make the DIP technology potentially attractive for a variety of biosensing applications. The DIP process could be monitored by measuring the open circuit potential of etched GaAs/AlGaAs biochips [5], however, more conveniently it is monitored with the photoluminescence (PL) effect induced in GaAs/AlGaAs nanoheterostructures $[6,7]$. The photocorrosion rate undergoes a drastic change when the photocorrosion front passes through a $\mathrm{GaAs} / \mathrm{AlGaAs}$ interface, resulting in the formation of a PL intensity maximum. This effect is modified by the interaction with negatively charged bacteria, which could result in a delayed position of PL maximum. The photocorrosion rate depends also on the concentration and nature of surface states that vary from sample-to-sample and results in fluctuations of the position of PL maxima. This affects the calibration error of a DIP biosensor.

The formation of an internal reference would require the removal of a biosensing architecture, typically employing alkanethiol self-assembled monolayers (SAM), from the selected area of a GaAs/AlGaAs biochip. This could be achieved by chemical etching in $\mathrm{HCl}$ [8] and $\mathrm{H}_{2} \mathrm{O}_{2}$ [9] solutions, or UV-assisted chemical etching [10] and selective area atomic layer deposition [11]. However, selective area microfabrication technique is a complicated processes requiring the application of photoresist-based lithography [12] that would not be acceptable for the realization of a low-cost biosensor. It has been demonstrated that hexadecanethiol (HDT) SAM desorb in vacuum from the GaAs surface at $180{ }^{\circ} \mathrm{C}$ [13]. Selected area heating of semiconductors to high temperatures could be achieved easily with IR lasers. For instance, it has been reported that a thermally isolated $10 \mathrm{~mm}$ x $10 \mathrm{~mm} \mathrm{GaAs} / \mathrm{Al}_{0.31} \mathrm{Ga}_{0.69} \mathrm{As}$ chip, coated with a $270 \mathrm{~nm}$ thick layer of $\mathrm{SiO}_{2}$, could be heated to $\sim 800{ }^{\circ} \mathrm{C}$ within 15 sec if irradiated with a continuous wave $(\mathrm{CW}) \mathrm{Nd}$ :YAG laser delivering power of $1 \mathrm{~W} / \mathrm{mm}^{2}$ to a 3-mm diameter spot [14]. Thus, this approach appears attractive for selective area desorption of SAM from the surface of biofunctionalized biochips. It is expected that the portion of a biochip with the laser created SAM-free zone would photocorrode at a significantly faster rate, typical of as-fabricated GaAs/AlGaAs nanoheterostructures.

The internal referencing concept has been investigated for optical and MEMs biosensor systems, resulting in new detection limits [15-17]. For instance, to address environmental and nonspecific effects, a label free Micro RNA cantilever has been employed as an internal reference for detection of cancer in cell lysates [18]. In this paper, we report on an IR laser-based process of selective area desorption of SAM from the surface of GaAs/AlGaAs biochips investigated for the formation of an internal reference in the operation of a DIP biosensor. 


\section{Experimental section}

\subsection{Fabrication of self-assembled monolayers}

Prior to deposition of 16-mercaptohexadecanoic acid (MHDA) SAM, $2 \mathrm{~mm}$ × $2 \mathrm{~mm}$ double sided polished samples of the GaAs/AlGaAs nanoheterostructures (Wafer D3422, CMC microsystems) were cleaned following the procedure described elsewhere [9]. After ultrasonication with OptiClear, acetone and isopropanol during $5 \mathrm{~min}$ in each solution, the samples were dried with ultra high-purity 5.0 grade nitrogen $(99.999 \%)$ and etched in ammonium hydroxide ( $28 \%$ aqueous solution of $\mathrm{NH}_{4} \mathrm{OH}$ ) for 2 min to remove native oxides from the GaAs surface. Then, the samples were rinsed with degassed ethanol, and immediately incubated in MHDA solution ( $1 \mathrm{mM}$, deoxygenated ethanol) for $20 \mathrm{~h}$ at room temperature and in darkness. A freshly cleaned sample was incubated in degassed ethanol for $20 \mathrm{~h}$ and used as background for FTIR measurements and PL analysis. After SAM formation, the samples were rinsed with degassed ethanol and ultrasonicated to remove any physisorbed thiol molecules from the surface. Finally, the samples were dried with nitrogen and stored in darkness under gas nitrogen atmosphere.

\subsection{Conventional RTA setup}

Initially, the desorption of SAMs from the GaAs surface was investigated in pristine conditions provided by the commercial Rapid Thermal Annealing setup (RTA, JIPELEC, JetFirst) as schematically illustrated in Figure 1. This setup comprises a bank of halogen lamps as a heat source operating up to $1200{ }^{\circ} \mathrm{C}$, and a supply of 4.3 grade oxygen $(99.993 \%)$ and 5.0 nitrogen $(99.999 \%)$ to create a desired controlled atmosphere $\left(30 \% \mathrm{O}_{2}\right.$ and $70 \% \mathrm{~N}_{2}$ was used in these experiments) maintained at a pressure of around 1040 mbar. The temperature was controlled with a thermocouple (K type) in contact with the backside of a silicon wafer used to support the investigated samples.

\subsection{Light Emitting Diode RTA setup}

The light emitting diode (LED) RTA setup, as schematically shown in Figure 2, was used for the annealing experiments carried out in a normal atmospheric environment. The setup consists of a CW LED emitting up to $150 \mathrm{~W}$ of the 980 $\mathrm{nm}$ radiation. The LED emission is coupled with the fiber whose output faces a 2-inch diameter silicon wafer used to support processed samples. The fiber numerical aperture of 0.22 allows to heat the $\mathrm{Si}$ wafer in excess of $500{ }^{\circ} \mathrm{C}$ [19]. The temperature of processed GaAs/AlGaAs samples was measured with a thermocouple ( $\mathrm{K}$ type) remaining in contact with the top surface of a sample. The temperature measurements and control were carried out with a dedicated Datalogger. A PM30 sensor connected to Molectron EPM2000

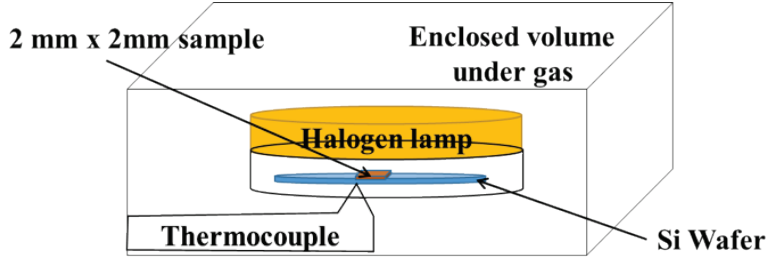

Fig. 1 Schematic illustration of the conventional RTA setup (JIPELEC). power meter was used to measure the LED output power. To induce a temperature of around $400{ }^{\circ} \mathrm{C}$, the LED was driven with a current of $\sim 16$ A allowing to generate output power of up to $\sim 22 \mathrm{~W}$. For the $30 \mathrm{~mm}$ distance between the output fiber and the silicon wafer, these conditions allowed to irradiate the silicon wafer area of $\sim 13 \mathrm{~mm}$ in diameter with estimated power density of $0.15 \mathrm{~W} / \mathrm{mm}^{2}$.

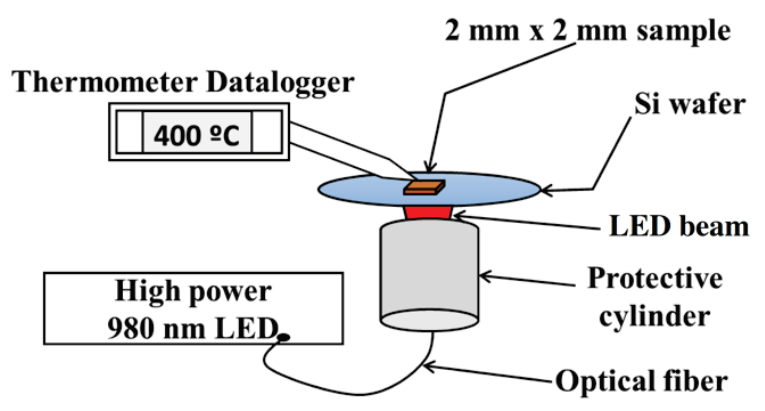

Fig. 2 Schematic illustration of the $980 \mathrm{~nm}$ LED RTA setup.

\subsection{Nd:YAG Laser RTA setup}

An Nd:YAG laser setup was used for selective area irradiation of MHDA SAM coated samples. A schematic illustration of the setup is shown in Figure 3 a). It consists of a CW Nd:YAG TEM 00 laser emitting at $1064 \mathrm{~nm}$ and delivering power of up to $30 \mathrm{~W}$. To deliver 1 to $12 \mathrm{~W}$ power to the sample surface, a 50/50 beam splitter was used, which allowed to avoid running a noisy laser operating in the vicinity of its operational threshold.

In order to minimize the potential contamination of processed samples with graphite released impurities, the samples were installed on a $6 \mathrm{~mm}$ x $5 \mathrm{~mm}$ Si substrate mounted above a $4 \mathrm{~mm} \times 4 \mathrm{~mm}$ opening in the graphite wafer that was suspended by the needle-tip fused silica rods. A Gaussian beam of the laser was focused to a $500 \mu \mathrm{m}$ diameter spot on the surface of GaAs/AlGaAs biochips. The profile of a laser beam as obtained with the Coherent beam profiler (LaserCam-HR II) employing a $1064 \mathrm{~nm}$ filter (MYQ-511064/HPTF6279, Optics for Research) is shown in Figure $3 \mathrm{~b}$. The laser spot diameter was calculated with the associated "BeamView-USB" software for the beam intensity reduced to $1 / \mathrm{e}^{2}$ of the peak value. The focal plane was at 250 $\mathrm{mm}$ from the employed lens.

\subsection{Photoluminescence measurements}

The photoluminescence (PL) intensity monitored DIP of $\mathrm{GaAs} / \mathrm{AlGaAs}$ nanoheterostructures was performed in a custom designed Quantum Semiconductor Photonic Biosensor (QSPB) Reader-2 [1, 4, 5]. The QSPB-2 Reader consists of an LED emitting at $625 \mathrm{~nm}$ for exciting GaAs/AlGaAs samples, an $812 \mathrm{~nm}$ long pass filter to collect lect PL data, and a CCD camera. The samples were mouned in a flow cell filled with $0.1 \mathrm{X}$ phosphate buffered saline (PBS) solution, or with PBS suspension of bacteria, both flown at $0.04 \mathrm{~mL} / \mathrm{min}$. The DIP process was induced with a $14 \mathrm{~mW} / \mathrm{cm}^{2}$ radiation $(1.8 \mathrm{~s}$ irradiation in each $15 \mathrm{~s}$ period, and PL was simultaneously monitored by collecting images of processed samples using a CCD camera. 


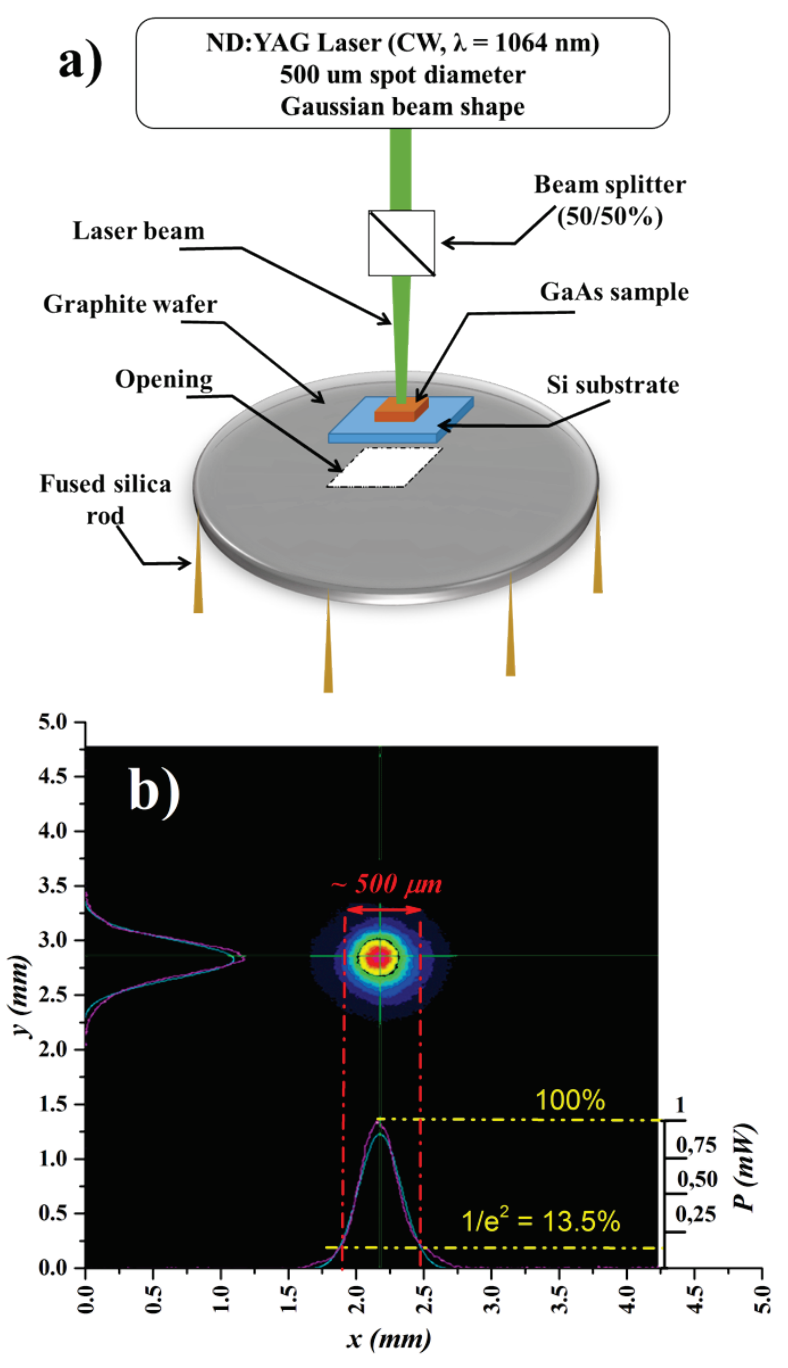

Fig. 3 Schematic illustration of the Nd:YAG laser RTA setup (a), and $2 \mathrm{D}$ profile of the laser beam employed for desorption of SAM (b).

\subsection{FTIR measurements}

Spectra of SAM formed on the GaAs surface were analyzed by Fourier transform IR (FTIR) transmission spectroscopy of asymmetric and symmetric $\mathrm{CH}_{2}$ stretch vibrations of alkane chains. The measurements were carried out with Bruker Vertex 70v vacuum spectrometer equipped with a RockSolid interferometer and a Globar IR source covering spectral range between 6000 and $10 \mathrm{~cm}^{-1}$. The signal was collected with a liquid-nitrogen cooled mercury cadmium telluride (MCT) IR detector. The spectral resolution and the numerical aperture were set to $4 \mathrm{~cm}^{-1}$ and $1.5 \mathrm{~mm}$, respectively. All measurements were carried out 5 min after evacuation of the FTIR chamber. The spectra were averaged over 1000 scans and subtracted from the spectrum of a freshly etched GaAs/AlGaAs sample considered as a background. MHDA SAM coated GaAs samples were characterized before and after thermal treatment designed to evaluate the effect of thermally induced desorption.

\subsection{Biofunctionalization protocol}

The specific capture of bacteria by GaAs/AlGaAs biosensors is achieved with an architecture comprising SAM and antibodies (Abs) as schematically illustrated in Figure 4.
To capture Abs, the samples were incubated for $30 \mathrm{~min}$ in an EDC-NHS solution (0.4 M-0.1 M). This allowed for activation of the $\mathrm{COOH}$ terminal group of MHDA, as illustrated in Figure 5. Following washing with deionized (DI) water, the sample were exposed to E. coli Abs that bind through their amine group to the activated $\mathrm{COOH}$. To saturate the unreacted $\mathrm{COOH}$ groups, the chips were exposed for 1 hour at $\mathrm{pH} 8$ in a $1 \mathrm{M}$ ethanolamine solution. Finally, the chips were washed with a $1 \%$ Tween 20 solution in PBS, to remove the non-covalently bound Abs [9]. Before optical microscopy measurements, the chips were washed again with DI water and dried under nitrogen to remove the remaining Tween solution.

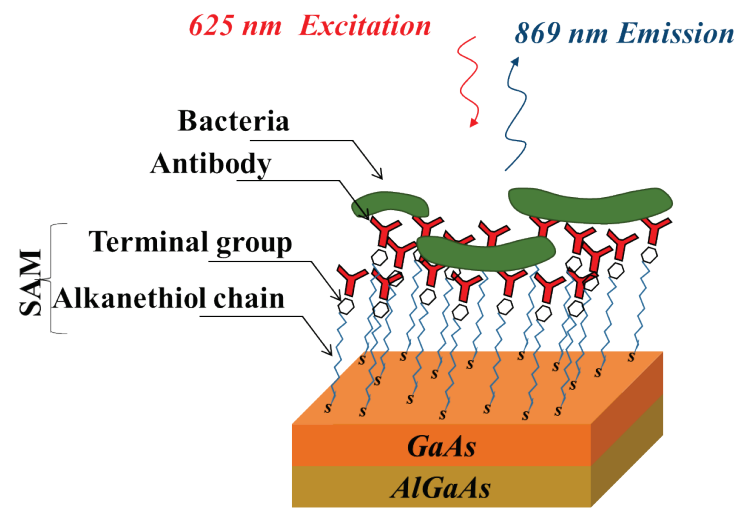

Fig. 4 Schematic illustration of the GaAs/AlGaAs biochip architecture with the excitation and emission wavelengths.

\section{Experimental results and discussion}

\subsection{RTA under controlled gas atmosphere}

The FTIR spectra shown in Figure 6 illustrate the quality of MHDA SAM coated GaAs samples before and after RTA annealing at $440{ }^{\circ} \mathrm{C}$. The formation of MHDA SAM on GaAs surface is confirmed by absorbance spectra of the asymmetric and symmetric $\mathrm{CH}_{2}$ stretch vibration modes at $2920 \mathrm{~cm}^{-1}$ and $2850 \mathrm{~cm}^{-1}$, respectively [20, 21]. It can be seen that the maximum absorbance intensity of the asymmetric stretching mode is about $1.3 \times 10^{-3}$ with a full widthat-half-maximum (FWHM) of $22 \mathrm{~cm}^{-1}$, which suggests the formation of a well-ordered SAM with the quality comparable to the best MHDA SAM reported in the literature [22]. In contrast, the spectra of the samples annealed for 10 and $15 \mathrm{~s}$ at $440{ }^{\circ} \mathrm{C}$ show the absence of $\mathrm{CH}_{2}$ features, consistent with a desorption of the MHDA SAM under these conditions. Figure 7 shows PL runs of DIP fresh (uncoated and non-annealed) and RTA samples. A weak PL intensity maximum is observed at $15 \mathrm{~min}$ for the uncoated and non-annealed sample, while the MHDA SAM coated and non-annealed sample revealed a broad PL maximum formed at $35 \mathrm{~min}$. This behaviour is consistent with the photocorrosion of similar nanoheterostructures reported elsewhere $[6,2,24]$. The PL intensities of the annealed samples evolve much slower and do not reveal formation of maxima within the $60 \mathrm{~min}$ DIP process. These results are consistent with desorption of MHDA SAM and accumulation of the DIP delaying residual products (oxides) on the surface of samples processed at $440{ }^{\circ} \mathrm{C}$ [6]. 


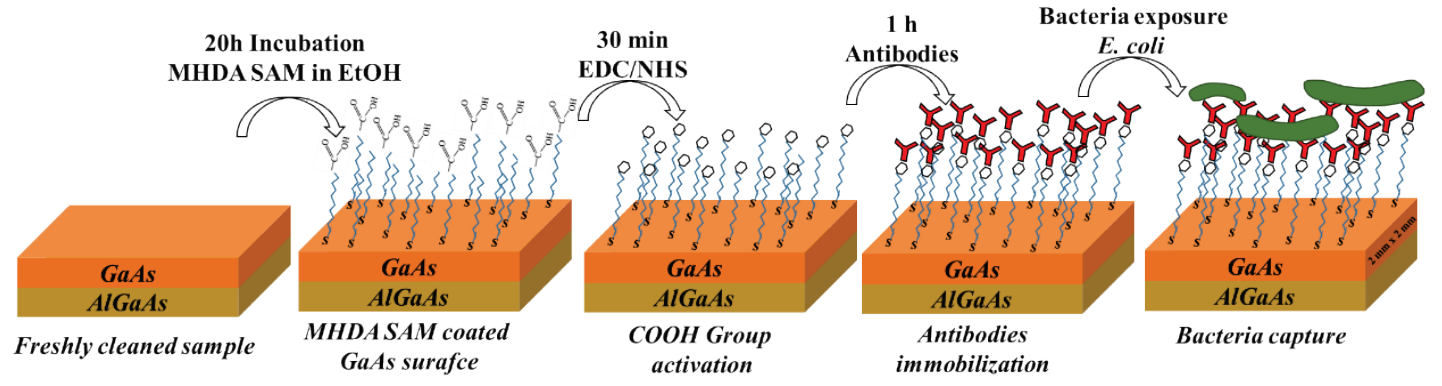

Fig. 5 Biofunctionalization scheme of a GaAs/AlGaAs biochip designed for the capture of bacteria.

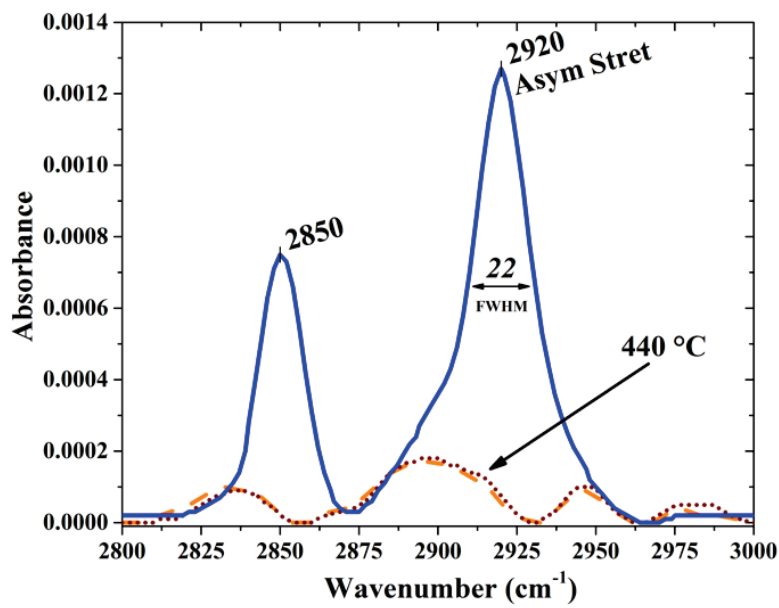

Fig. 6 FTIR absorbance spectra of an intact MHDA SAM coated $\mathrm{GaAs} / \mathrm{AlGaAs}$ sample (blue), and of similar samples annealed at $440{ }^{\circ} \mathrm{C}$ for $10 \mathrm{~s}$ (dash) and $15 \mathrm{~s}$ (dotted).

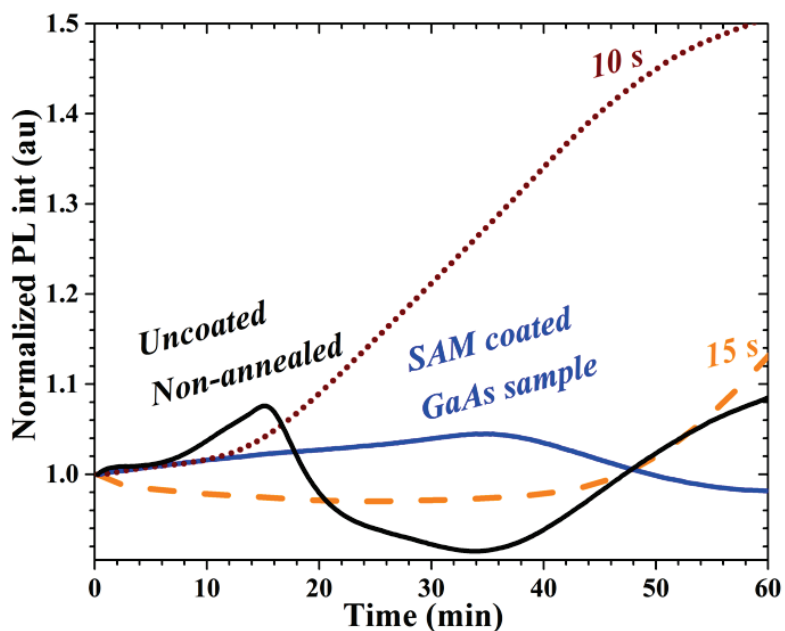

Fig. 7 PL monitored DIP of a fresh GaAs/AlGaAs sample (black), as-fabricated MHDA SAM coated sample (blue), and MHDA SAM coated samples annealed at $440{ }^{\circ} \mathrm{C}$ for $10 \mathrm{~s}$ (brown-dotted) and $15 \mathrm{~s}$ (yellow-dash).

The FTIR absorbance spectra of the samples annealed at lower temperatures are shown in Figure 8. It can be seen that while the annealing at $400{ }^{\circ} \mathrm{C}$ for $15 \mathrm{~s}$ leads to a similar result as that observed for the processing temperature of $440{ }^{\circ} \mathrm{C}$, the annealing at 360 and $320^{\circ} \mathrm{C}$ only partially removes SAM as illustrated by the weak absorbance spectra of $\mathrm{CH}_{2}$ stretch vibration features. The 3 to $5 \mathrm{~cm}^{-1}$ shifts of these features to higher energies suggest the presence of less organized SAM. These results are consistent with the observations made by

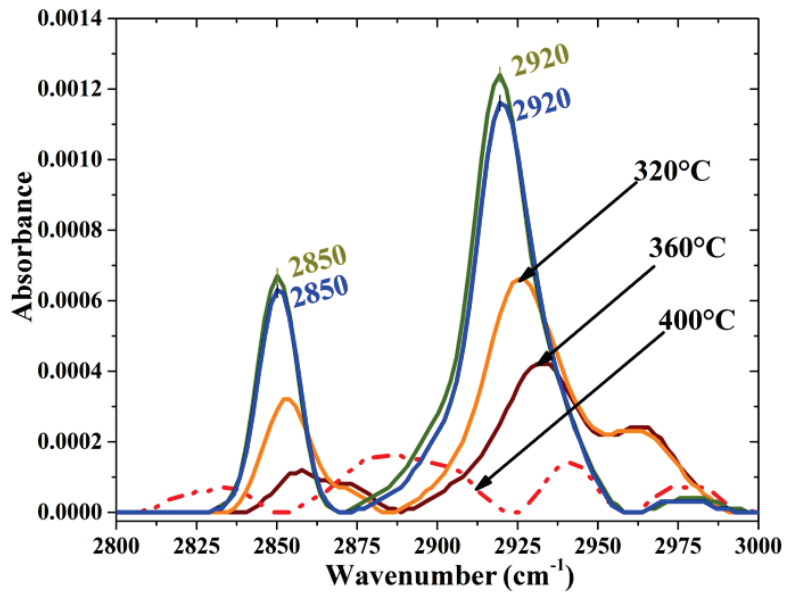

Fig. 8 FTIR absorbance spectra of as-fabricated GaAs/AlGaAs samples coated with MHDA SAM (solid blue) and annealed at $320^{\circ} \mathrm{C}$ (solid yellow), $360^{\circ} \mathrm{C}$ (solid brown) and $400{ }^{\circ} \mathrm{C}$ (dot-dash red). Notice that ammonia processing of as-fabricated sample (solid green) does not affect the quality of SAM.

Huang et al., [25] who reported a partial dissociation of short alkanethiol SAM from the GaAs surface in vacuum at $247^{\circ} \mathrm{C}$, and their complete decomposition at $392^{\circ} \mathrm{C}$.

Figure 9 compares PL plots collected during DIP of a freshly cleaned GaAs/AlGaAs sample, an MHDA SAM coated sample and an MHDA SAM coated sample annealed at $400{ }^{\circ} \mathrm{C}$. A delayed position of the PL intensity maximum from 15 to $35 \mathrm{~min}$ is consistent with the presence of MHDA SAM that slows down the photocorrosion process $[6,2,24]$. The annealing of an MHDA SAM coated sample at $400{ }^{\circ} \mathrm{C}$ removes SAM from its surface, and the position of a related PL intensity maximum is observed at $23 \mathrm{~min}$, i.e., delayed by $8 \mathrm{~min}$ in comparison to that maximum observed for the as-fabricated (uncoated) sample. It has been known that high-temperature processing of GaAs leads to surface accumulation of As- and Ga-oxides [26]. While As-oxides are relatively easily dissolvable in water [27], Ga-oxides are etchable, e.g., in ammonium hydroxide [28]. To verify if such oxides have contributed to the deceleration of the DIP process, the $400{ }^{\circ} \mathrm{C}$ annealed sample was subjected to a 2-min exposure of an aqueous solution of ammonium hydrox ide $(28 \%)$. The associated PL plot in Figure 9 exhibits a maximum at $\sim 16$ min that coincides well with the $15-\mathrm{min}$ maximum revealed for the uncoated and non-annealed sample. This result suggests that the ammonia post treatment of annealed samples allows to remove excessive oxides, 


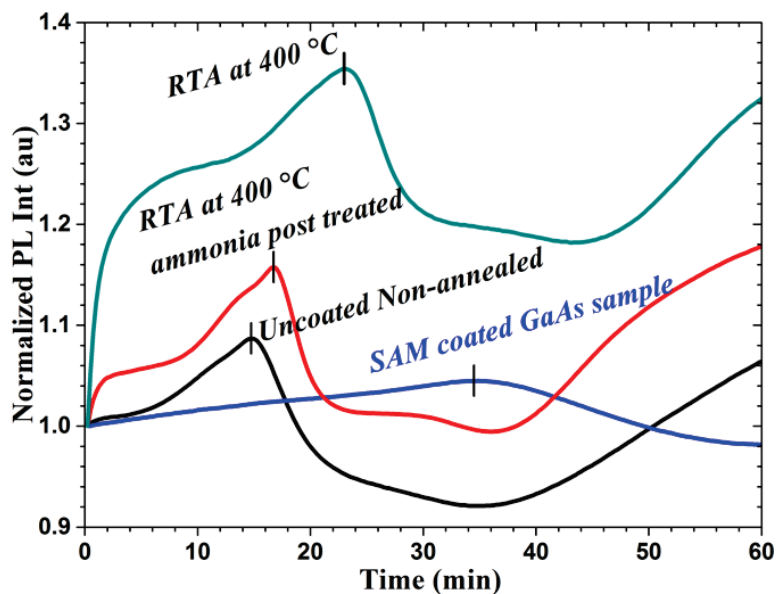

Fig. 9 PL intensity plots of DIP uncoated (black) and MHDA SAM coated (blue) samples compared to the $400{ }^{\circ} \mathrm{C}$ annealed MHDA SAM coated sample (turquoise). The photocorrosion of ammonia processed MHDA SAM coted sample annealed at $400{ }^{\circ} \mathrm{C}$ (red) proceeds similarly to that of the as-fabricated sample.

formed during annealing, and fabricate attractive samples with a built-in reference. We have also verified if ammonia processing of MHDA SAM coated sample is not detrimental to the quality of the formed SAM and allows preserving its integrity. Indeed, as shown in Figure 8, the FTIR spectrum of an ammonia processed sample for 2 min (green) is practically undistinguishable from that of the original, non-processed sample (blue).

\subsection{LED RTA in atmospheric environment}

The FTIR absorbance spectra of samples processed by LED-RTA for $15 \mathrm{~s}$ at 300,350 and $400{ }^{\circ} \mathrm{C}$ are shown in Figure 10. The results suggest that the desorption of SAM takes place for the samples annealed at 400 and $350^{\circ} \mathrm{C}$, consistent with the disappearance of the absorbance spectra corresponding to the $\mathrm{CH}_{2}$ vibrations. A sample annealed at $300{ }^{\circ} \mathrm{C}$ shows the presence of a partially removed SAM. Figure 11 shows the results of the PL monitored DIP process of as-fabricated and annealed at $350{ }^{\circ} \mathrm{C}$ samples treated in ammonia. The formation of PL maxima for the uncoated and SAM coated samples can be seen, respectively, at 15 and $35 \mathrm{~min}$, while the $350{ }^{\circ} \mathrm{C}$ annealed and ammonia post-processed samples show PL maxima at 20 and $23 \mathrm{~min}$. Slightly delayed PL maxima positions for the LED-RTA samples, in comparison to the PL maximum of the uncoated and non-annealed sample may be related to the surface presence of some oxides that were not etched with ammonia in this case.

\subsection{Bacteria immobilization on LED-RTA processed biochip}

Figure 12 compares scattering light optical images of an MHDA SAM coated sample functionalized with Abs and exposed for 1 hour to $E$. coli $\mathrm{PBS}$ suspension at $10^{9} \mathrm{CFU} / \mathrm{mL}$, followed by washing with DI water and drying under a flow of nitrogen (Fig. 12a), with that of a similar sample that was $15 \mathrm{~s}$ annealed at $350{ }^{\circ} \mathrm{C}$ before the exposure to the same $E$. coli suspension (Fig. 12b). The significant capture of bacteria, estimated at 2542 bacteria $/ \mathrm{mm}^{2}$, has been observed for the as-fabricated sample (Fig. 12 a). In contrast, the entire surface of the $2 \mathrm{~mm} \times 2 \mathrm{~mm}$ sample annealed at $350{ }^{\circ} \mathrm{C}$ did

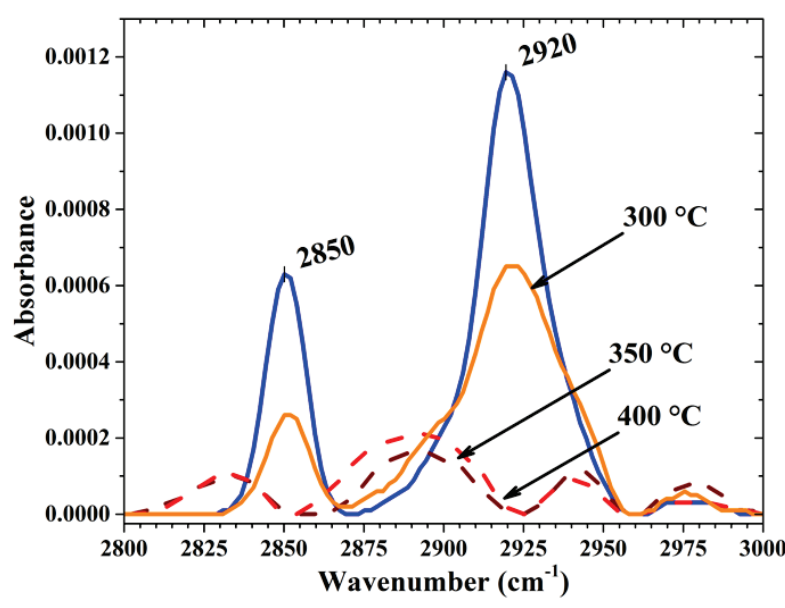

Fig. 10 FTIR absorbance spectra of as-fabricated GaAs/AlGaAs samples coated with MHDA SAM (blue) and annealed at $300^{\circ} \mathrm{C}$ (yellow), $350{ }^{\circ} \mathrm{C}$ (dashed red) and $400{ }^{\circ} \mathrm{C}$ (dashed brown).

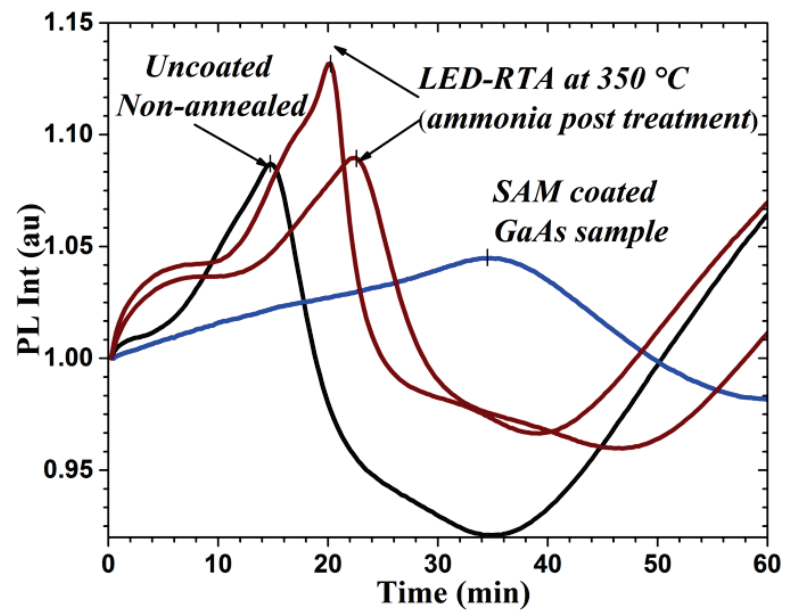

Fig. 11 PL intensity plots of DIP uncoated (black) and MHDA SAM coated (blue) samples compared to the plots of the MHDA SAM coated samples annealed for $15 \mathrm{~s}$ at $350{ }^{\circ} \mathrm{C}$ (brown).

not show a measurable presence of the captured bacteria. This result is consistent with the absence of Abs, but also with the absence of MHDA SAM that is expected to desorb under these conditions. It is relevant to mention that a chemically etched and non-functionalized surface of GaAs does not provide conditions favouring the capture of bacteria [29] Furthermore, the washing with the $1 \%$ Tween 20-PBS solution employed in this experiment, allowed to remove the non-specifically attached E. coli [30].

\subsection{Selective area functionalization by Nd:YAG Laser RTA}

\subsubsection{Irradiation at $43 \mathrm{~W} / \mathrm{mm}^{2}$ for $30 \mathrm{~s}$}

To desorb MHDA SAM from a selected area of the $\mathrm{GaAs} / \mathrm{AlGaAs}$ biochip, the irradiation was carried out with an Nd:YAG laser beam focused 1 to a $0.5 \mathrm{~mm}$ diameter spot. Figure 13 shows FTIR absorbance spectra of MHDA SAM coated GaAs surface before and after irradiation at 43 $\mathrm{W} / \mathrm{mm}^{2}$ for $30 \mathrm{~s}$. We have estimated that the maximum temperature induced under these conditions was $\sim 500{ }^{\circ} \mathrm{C}$ in the 

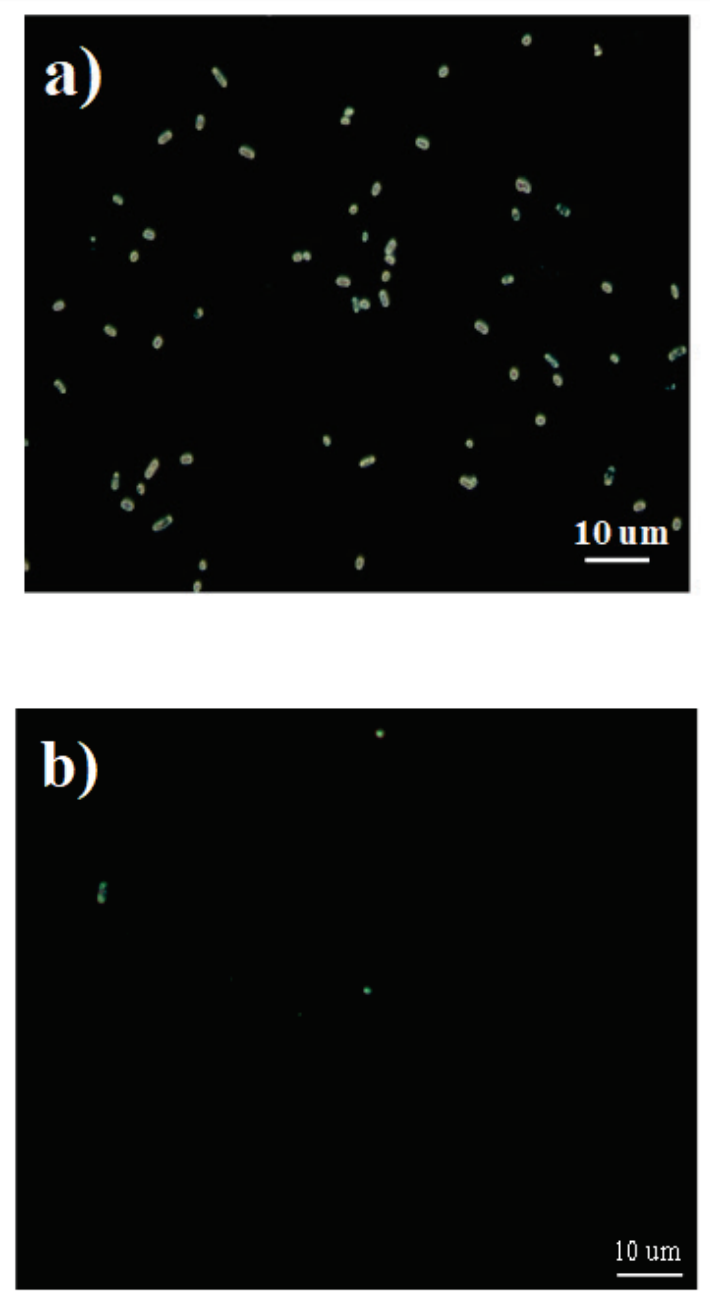

Fig. 12 Scattering light optical microscopy images of a) antibody biofunctionalized GaAs sample, and b) antibody biofunctionalized sample following LED-RTA at $350{ }^{\circ} \mathrm{C}$ for $15 \mathrm{~s}$. In both cases, the samples were exposed to $E$. coli $\mathrm{PBS}$ suspension at $10^{9} \mathrm{CFU} / \mathrm{mL}$ for 1 hour.

center of a laser spot. Notice that the irradiation of a thermally isolated $10 \mathrm{~mm} \times 10 \mathrm{~mm}$ GaAs sample with the same laser delivering power of $1 \mathrm{~W} / \mathrm{mm}^{2}$ allowed to induce the temperature of $\sim 820^{\circ} \mathrm{C}$ in the center of a $3 \mathrm{~mm}$ diameter spot [14]. However, the sample in that case was double sided coated with a $270 \mathrm{~nm}$ thick layer of $\mathrm{SiO}_{2}$ that was acting as an anti-reflection and allowed decreasing the reflected power from 34 to $10 \%$ of the IR radiation [31-33].

The spectra shown in Figure 13 were collected from a 1-mm diameter surface area defined by the applied contact mask. A weak intensity spectrum, with poorly defined positions of the asymetric and symetric stretch vibrations peaks characterizes the Laser-RTA sample. Given that some contribution to this spectrum originated from the nonirradiated surface of the sample, this result suggests the complete desorption of MHDA SAM in the center of the laser irradiated spot. Following the irradiation, the sample was exposed for 2 min in ammonia and its PL-monitored DIP revealed a PL intensity maximum at $21 \mathrm{~min}$, as illustrstes Fig. 14. In contrast, several PL scans of the non-irradiated areas exhibit a slower photocorrosion rate with PL maxima revealed at $\sim 30 \mathrm{~min}$, which is in a reasonable agreement with the position of such maxima revealed for other,

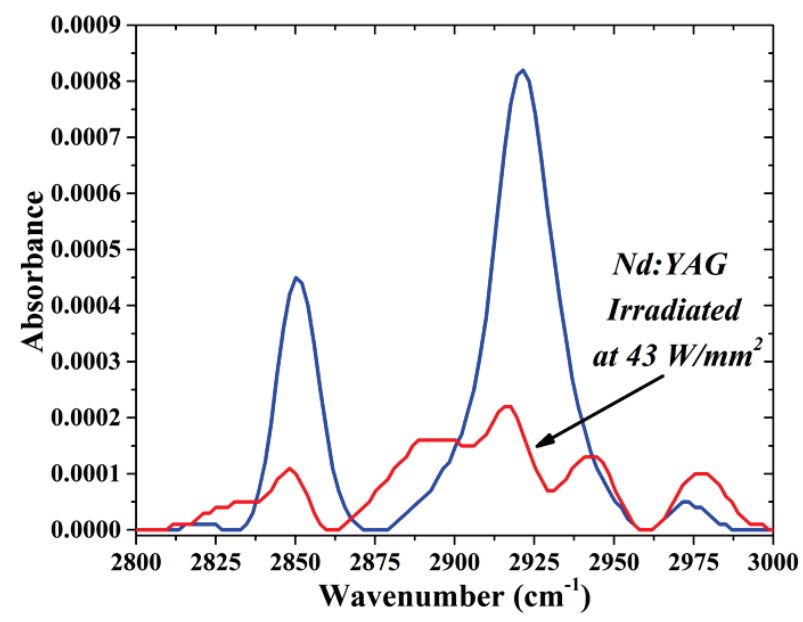

Fig. 13 FTIR absorbance spectra of as-fabricated GaAs/AlGaAs sample coated with MHDA SAM (blue), and a sample irradiated with an Nd:YAG laser at 43 $\mathrm{W} / \mathrm{mm}^{2}$ (red).

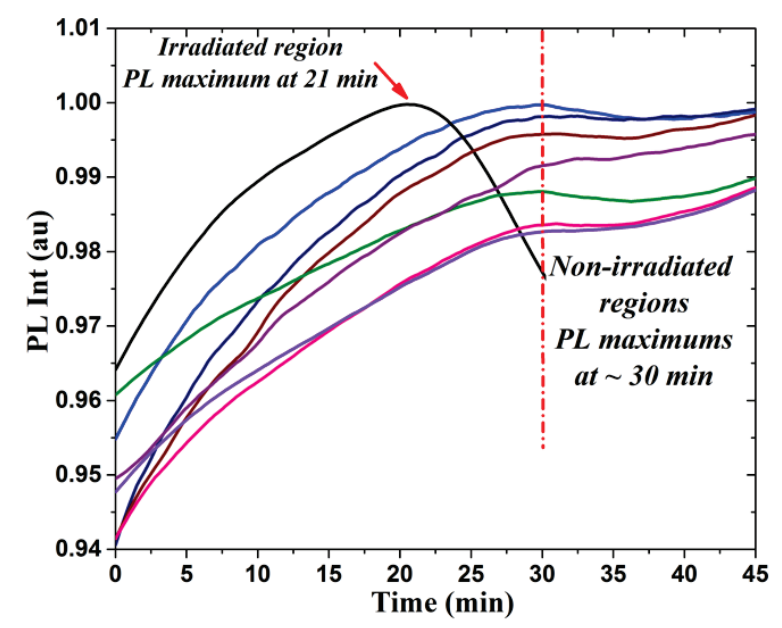

Fig. 14 PL intensity plots of a DIP sample with an irradiated region (black) showing faster photocorrosion rate than those from MHDA SAM coated regions (colored).

non-annealed MHDA SAM coated samples (see also Figure 11).

Figure 15 shows a PL intensity map of an Nd:YAG RTA sample that was DIP for $21 \mathrm{~min}$. The background PL signal was removed from this map to illustrate more clearly the laser irradiated area of $\sim 500 \mu \mathrm{m}$ in diameter. Notice that the DIP process is sensitive to the presence of surface imperfections that could also act as non-radiative recombination centers generated during the Laser RTA step. This seems to be the reason of a slight deviation in the uniformity of the PL intensity of the laser annealed area compared to the $\mathrm{TM}_{00}$ mode of the laser beam (see Fig. 3) used for the annealing. The inspection of PL intensity plots collected for different DIP non-annealed regions of this SAM coated sample revealed distinctive maxima located at $\sim 30 \mathrm{~min}$, as illustrated in Figure 14.

3.4.2 Bacteria capture on Nd:YAG-RTA biochip An Nd:YAG laser irradiated MHDA SAM coated 


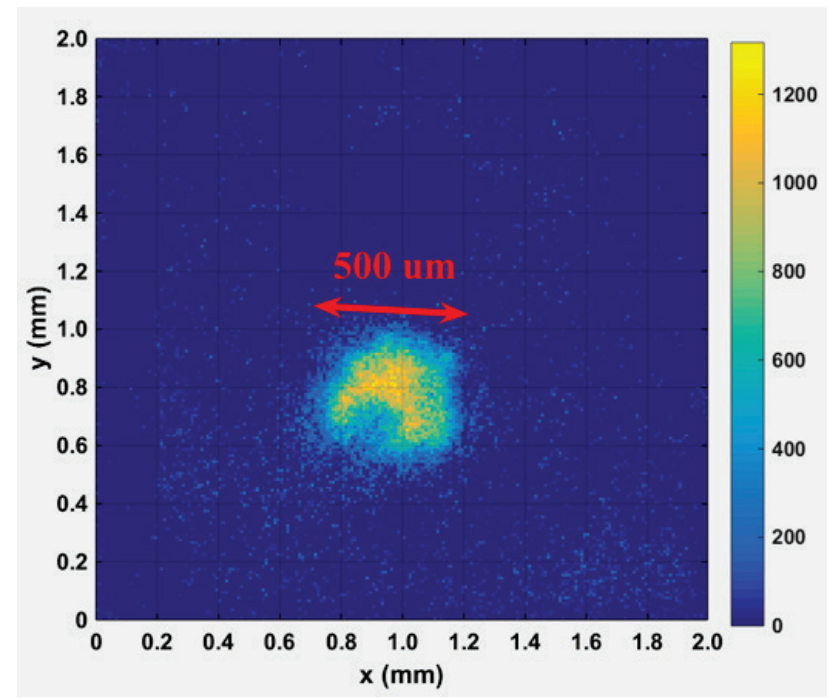

Fig. 15 Photoluminescence intensity map of an MHDA SAM coated sample that was $\mathrm{Nd}$ :YAG RTA at $\sim 500{ }^{\circ} \mathrm{C}$ for $30 \mathrm{~s}$ and, subsequently, photocorroded for $21 \mathrm{~min}$.

GaAs sample was post-treated in ammonia, biofunctionalized with Abs (achieved in the non-annealed portion of the sample) and immediately exposed to E. coli suspension at $10^{9} \mathrm{CFU} / \mathrm{mL}$. Following this step, the sample was rinsed with DI water to remove weakly bound biomolecules and bacteria from the surface of the biochip. Figure 16 a) shows an optical image of the sample covered with bacteria, except in the laser irradiated area identified with a circle of $\sim 350$ $\mu \mathrm{m}$ in diameter. Although some bacteria could be identified in the laser annealed region, their surface density is negligible compared to that in the non-annealed region. Clearly, some non-specific attachement of bacteria is expected on many surfaces exposed to highly concentrated bacterial suspensions, such as $10^{9} \mathrm{CFU} / \mathrm{mL}$ employed in this experiment. Nevertheless, we argue that this result is consistent with the absence of Abs in the laser processed spot. While the bare surface of GaAs does not immobilize bacteria, the presence of EDC-NHS processed MHDA SAM would be required to immobilize Abs. Figure 16 b) shows a high-resolution image of the non-annealed fragment of the sample. The presence of uniformly distributed bacteria can be easily identified in that image. We estimated that the concentration of bacteria captured in this case is at 2585 bacteria $/ \mathrm{mm}^{2}$, which is comparable to the concentration of $E$. coli captured on the surface of a non-annealed bio-functionalized GaAs sample (see Fig. 12 a). Thus, this result confirms the feasibility of a concept of selective area desorption of MHDA SAM from GaAs for the realization of a DIP GaAs/AlGaAs biochip with an internal reference.

\section{Conclusion}

We have investigated selective area functionalization of GaAs/AlGaAs biochips by employing the effect of thermal desorption of MHDA SAM. The conventional RTA experiments and LED-based RTA of MHDA SAM coated GaAs samples allowed to determine conditions for annealing with a CW Nd:YAG laser operating at $1064 \mathrm{~nm}$, designed for selective area desorption of SAM. Following the exposure to a bacterial suspension at $10^{9} \mathrm{CFU} / \mathrm{mL}$, the EDC-NHS activated MHDA SAM functionalized with Abs allowed
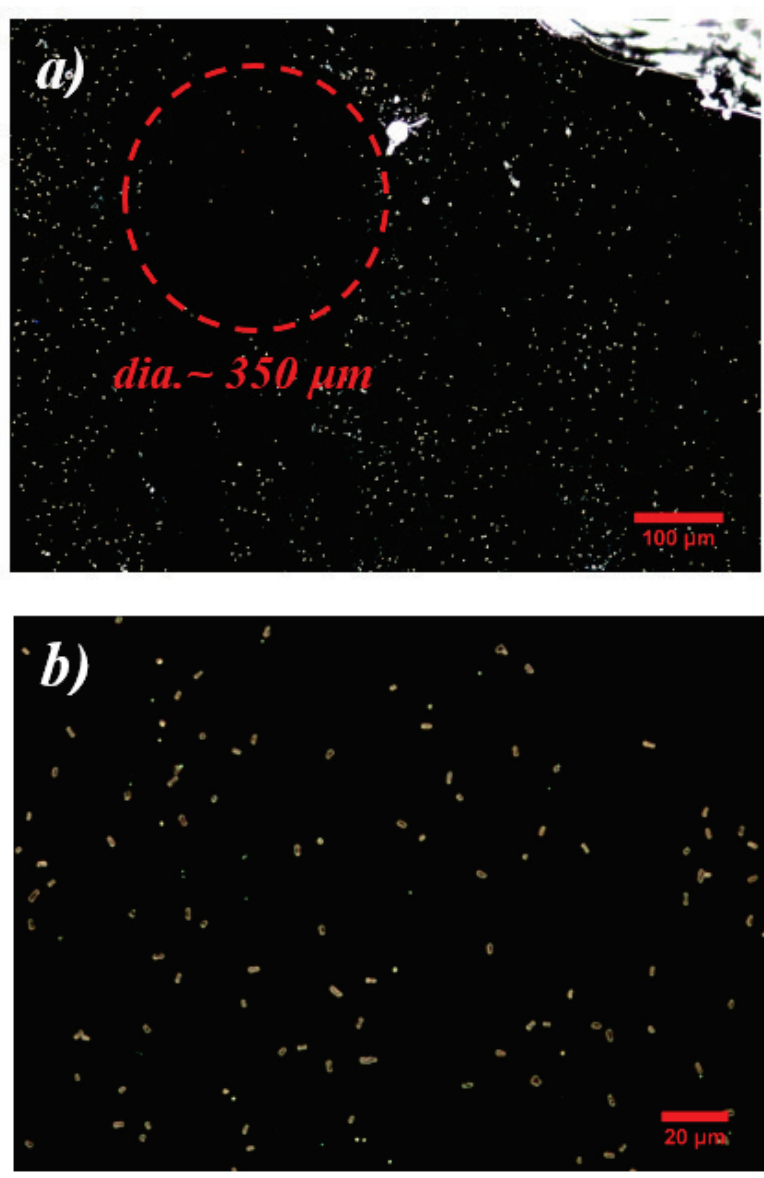

Fig. 16 Low-magnification (a) and enhanced magnification (b) scattering light optical microscopy images of a bio-functionalized GaAs sample following selected area Nd:YAG laser irradiation and exposure to $E$. coli at $10^{9} \mathrm{CFU} / \mathrm{mL}$. The laser irradiated area is identified with a circle.

capturing bacteria at $\sim 2500$ bacteria $/ \mathrm{mm}^{2}$. The irradiation of an Abs functionalized biochip with a $\mathrm{TEM}_{00}$ beam of the $\mathrm{Nd}$ :YAG laser delivering $\sim 43 \mathrm{~W} / \mathrm{mm}^{2}$ radiation for $30 \mathrm{~s}$ to a $2 \mathrm{~mm} \times 2 \mathrm{~mm}$ sample, allowed to fabricate a $350 \mu \mathrm{m}$ diameter SAM-free and, thus, Abs-free zone. The SAM-free zone photocorroded at a rate comparable to that of a freshly cleaned, uncoated chip. The similar photocorrosion rate was also observed for the SAM-free sample fabricated by LEDRTA at $350{ }^{\circ} \mathrm{C}$ for $15 \mathrm{~s}$. Our results demonstrate the feasibility of employing the concept of selective area desorption of MHDA SAM from GaAs for the realization of a DIP GaAs/AlGaAs biochip with an internal reference. The currently available low-cost IR LED sources delivering power densities sufficient to induce spot annealing of GaAs at 350$400{ }^{\circ} \mathrm{C}$ offer the potential for the realization of advanced DIP biosensors with internal references.

\section{Acknowledgements}

This project was supported by the Natural Sciences and Engineering Research Council of Canada (NSERC) Discovery Grant (RGPIN-2015-04448) and the NSERC Strategic Partnership Grant (SPG-2016-494057).

\section{References}

[1] E. Nazemi, W. M. Hassen, E. H. Frost, and J. J. Dubowski: Biosens. Bioelectron., 93, (2016) 234. 
[2] E. Nazemi, S. Aithal, W. M. Hassen, E. H. Frost, and J. J. Dubowski: Sens. Actuators B: Chem., 207, (2015) 556-562.

[3] M. R. Aziziyan et al:: Sens. Actuators B: Chem., 304, (2020) 127007.

[4] M. R. Aziziyan, W. M. Hassen, D. Morris, E. H. Frost, and J. J. Dubowski: Biointerphases, 11, (2016) 019301.

[5] S. Aithal and J. J. Dubowski: Appl. Phys. Lett., 112, (2018) 153102.

[6] H. Sharma, K. Moumanis, and J. J. Dubowski: J. Phys. Chem. C, 120, (2016) 26129.

[7] S. Aithal, N. Liu, and J. J. Dubowski: J. Phys. D: Appl. Phys., 50, (2017) 035106.

[8] X. Ding and J.J. Dubowski: Proc. of SPIE on Photon Processing in Microelectronics and Photonics VI, 6458, (2007) 64581C.

[9] V. Lacour, C. Elie-Caille, T. Leblois, and J. J. Dubowski: Biointerphases, 11, (2016) 019302.

[10]L. Wu et al.: Chemical Physics Letters, 717, (2019) 152.

[11]X. Jiang and S. F. Bent: J. Phys. Chem. C, 113, (2009) 17613.

[12]K.-H. Lee et al.: Organic Electronics, 11, (2010) 748.

[13]D. Cuypers et al.: Chem. Mater., 28, (2016) 5689.

[14]R. Stanowski, O. Voznyy, and J. J. Dubowski: J. Laser Micro/Nanoeng., 1, (2006) 17.

[15]Y. Lin et al.: Anal. Chem, 91, (2019) 3717.

[16]S. Wang et al.: ACS Appl. Mater. Interfaces, 11, (2019) 1917.

[17]X. Wang et al.: Anal. Chem., 91, (2019) 12006.

[18] J. Duffy, F. Padovani, G. Brunetti, P. Noy, U. Certa, and M. Hegner: The Royal Society of Chemistry Nanoscale, 10, (2018) 12797.

[19]R. Stanowski and J. J. Dubowski: Appl. Phys. A, 94, (2008) 667.

[20]X. Huang, N. Liu, K. Moumanis, and J. J. Dubowski: J. Phys. Chem. C, 117, (2013) 15090.

[21]P. Mancheno-Posso and A. J. Muscat: Appl. Surf. Sci., 397, (2017) 1.

[22] X. H. Huang, N. Liu, K. Moumanis, and J. J. Dubowski: J. Phys. Chem. C, 117, (2013) 15090.

[24] M. R. Aziziyan, H. Sharma, and J. J. Dubowski: ACS Appl. Mater. Inter., 11, (2019) 17968.

[25] T. P. Huang, T. H. Lin, T. F. Teng, Y. H. Lai, and W. H. Hung: Surf. Sci., 603, (2009) 1244.

[26] C. D. Thurmond, G. P. Schwartz, G. W. Kammlott, and B. Schwartz: J. Electrochem. Soc., 127, (1980) 1366.

[27]G. P. Schwartz, G. J. Gualtieri, J. E. Griffiths, C. D. Thurmond, and B. Schwartz: J. Electrochem. Soc., 127, (1980) 2488.

[28] G. C. DeSalvo et al.: J. Electrochem. Soc., 143, (1996) 3652.

[29] M. A. Islam, W. M. Hassen, A. F. Tayabali, and J. J. Dubowski: Biochem. Eng. Journal, 154, (2020) 107435.

[30] C. Wu, J. Y. Lim, G. G. Fuller, and L. Cegelski: Langmuir, 29, (2013) 920.

[31]J. Grames et al.: Phys. Rev. Special Topics Accelerators and Beams, 14, (2011) 043501.

[32]R. E. Morrison: Phys. Rev., 124, (1961) 1314.

[33]X. Peng et al.: Phys. Rev. Appl., 12, (2019) 064002.

(Received: February 17, 2020, Accepted: July 26, 2020) 